\title{
Explicit inverses of Toeplitz and associated matrices
}

\author{
Murray Dow* \\ (Received 7 June 2000, revised 7 January 2003)
}

\begin{abstract}
We discuss Toeplitz and associated matrices which have simple explicit expressions for their inverses. We first review existing results and generalize these where possible, including matrices with hyperbolic and trigonometric elements. In $\S 2$ we discuss and generalize the Fiedler matrix. In $\S 3$ we give an analytic procedure for inverting any band Toeplitz matrix, in $\S 4$ we invert a tridiagonal Toeplitz matrix with modified corner elements.
\end{abstract}

\section{Contents}

1 Introduction and review

E186

*Supercomputer Facility, Australian National University, Canberra 0200, Australia. mailto:m.dow@anu.edu.au

${ }^{0}$ See http://anziamj . austms . org. au/V44/E019 for this article, (C) Austral. Mathematical Soc. 2003. Published January 23, 2003. ISSN 1446-8735 
1.1 Matrix 1: $c+d|i-j| \ldots \ldots \ldots \ldots$ E188

1.2 Matrix 2: $(-1)^{i-j}(c+d|i-j|) \ldots \ldots \ldots$ E189

1.3 KMS matrix: $\rho^{|i-j|} \ldots \ldots \ldots$. . . . . . . E189

1.4 Nonsymmetric KMS matrix . . . . . . . . . E190

1.5 Generalized KMS matrix: $\alpha+\beta \rho^{|i-j|} \ldots \ldots$ E191

1.6 Hyperbolic matrix: $\alpha \rho^{-|i-j|}+\beta \rho^{|i-j|} \ldots \ldots . . \quad$ E193

1.7 Trigonometric matrix: $\alpha \sin (\rho|i-j|)+\beta \cos (\rho|i-j|)$ E195

2 Fiedler's matrix: $c_{j}-c_{i}$

E196

2.1 Generalized nonsymmetric Fiedler's matrix: $d+p c_{i}+$ $q c_{j}$

E197

3 Inverse of band Toeplitz matrix

E199

3.1 Example: General tridiagonal matrix . . . . . . E202

3.2 Example: third order difference operator matrix . E205

3.3 Example: fourth order difference operator matrix E206

4 Tridiagonal matrix with modified corner elements E208

5 Conclusion

E211

References

E211

\section{Introduction and review}

Toeplitz matrices were originally studied by Toeplitz [18, 29] who called the related quadratic form an $L$-form. A Toeplitz matrix is of the form $A_{i j}=c_{i-j}$ with $c_{-m}$ the complex conjugate of $c_{m}$, and they occur in many fields $[13,22]$. Here we report the results of our search for real Toeplitz matrices with simple explicit inverses.

The matrices in this paper occur in many applications, and they 
are useful as test matrices for numerical routines. Band matrices in particular occur frequently in linear equations; however, solution via a Gaussian elimination method is usually preferable to using a dense inverse, except when only a few elements of the solution are required.

For example when solving the $n$ linear equations $A x=b$, where $A$ is the band matrix in $\S 3.3$, but only require $x_{1}$, we have the exact

$$
x_{1}=\frac{1}{(n+2)(n+3)} \sum_{j=1}^{n} j(n+1-j)(n+2-j) b_{j}
$$

which is $5 n+2$ flops, compared to about $16 n$ flops for Gaussian elimination.

There are times when an explicit inverse is required; for example the determinant can usually be found once the inverse is known, as is done in some of the proofs in this paper. See also $[2,7,20]$. Portions of explicit inverses can also be used as pre-conditioners for the conjugate gradient method [8].

In section $\S 1$ we review existing results and generalize these, notably finding the hyperbolic and trigonometric matrices, and in $\S 2$ we generalize Fiedler's matrix [28]. While Fiedler's matrix is not in general a Toeplitz matrix, it is closely related to a Toeplitz matrix; for example its inverse has the same sparsity pattern as many of the matrices in $\S 1$.

In $\S 3$ we give a simple method for finding the inverse of a band Toeplitz matrix, which differs from Rozsa's [24] approach. We use these results to derive the inverse of a tridiagonal Toeplitz matrix, taking care to consider all possible values of the diagonals. These methods can be used to find the eigenvalues of these matrices (or an expression proportional to the characteristic polynomial); however the algebra is prohibitive except for very small bandwidth. Other 
methods, for example solving the recurrence relation in $x$ that is implicit in the eigenvalue equation $A x-\lambda x=0$ [19] or via determinants $[9,26]$, provide a more direct way to the characteristic polynomial. We find that the solutions of the recurrence (4) provide 'basis functions' for the elements of $A^{-1}$.

In $\S 4$ we illustrate the flexibility of this approach by deriving the inverse of a tridiagonal matrix with constant diagonals, but with modified corner elements.

In this paper we denote the order of the matrix $A$ by $n$. We begin by quoting examples from the literature.

\subsection{Matrix 1: $c+d|i-j|$}

In [28] the inverse of the matrix $A_{i j}=|i-j|, i, j=1, \ldots, n$ was given; we generalize this $(n>2)$ to the matrix:

$$
A_{i j}= \begin{cases}c+d_{1}|i-j|, \quad i \leq j, & i, j=1, \ldots, n \\ c+d_{2}|i-j|, & i \geq j, \quad i, j=1, \ldots, n\end{cases}
$$

which has the inverse

$$
A^{-1}=\frac{1}{d_{1}+d_{2}}\left[\begin{array}{rrrrrr}
-\xi_{n-1} / \xi_{n} & 1 & 0 & \cdots & 0 & d_{1}^{2} / \xi_{n} \\
1 & -2 & 1 & 0 & \cdots & 0 \\
0 & 1 & -2 & 1 & 0 & 0 \\
& & \ddots & \ddots & \ddots & \\
0 & \cdots & 0 & 1 & -2 & 1 \\
d_{2}^{2} / \xi_{n} & 0 & \cdots & 0 & 1 & -\xi_{n-1} / \xi_{n}
\end{array}\right]
$$

where $\xi_{n}=c\left(d_{1}+d_{2}\right)+d_{1} d_{2}(n-1)$ (see also [12, pp31,51] and [27]).

We shall see this sparsity pattern several times in this paper, namely tridiagonal with constant diagonals except for the corner 
elements, and we shall see that this matrix is a particular case of the generalized Fiedler matrix discussed in $\S 2$. The determinant is $|A|=-(-1)^{n}\left(d_{1}+d_{2}\right)^{n-2} \xi_{n}$.

A band matrix form of this, namely $A_{i j}=k-|i-j|$ for $|i-j|<k$, zero otherwise, was inverted in [21].

\subsection{Matrix 2: $(-1)^{i-j}(c+d|i-j|)$}

The corresponding result, in which the three diagonals of the inverse have the same sign is of interest $(n>2)$ :

$$
A_{i j}= \begin{cases}(-1)^{i-j}\left(c+d_{1}|i-j|\right), & i \leq j, \quad i, j=1, \ldots, n \\ (-1)^{i-j}\left(c+d_{2}|i-j|\right), & i \geq j, \quad i, j=1, \ldots, n\end{cases}
$$

has the inverse

$$
A^{-1}=-\frac{1}{d_{1}+d_{2}}\left[\begin{array}{rrrrrr}
\xi_{n-1} / \xi_{n} & 1 & 0 & \cdots & 0 & (-)^{n} d_{1}^{2} / \xi_{n} \\
1 & 2 & 1 & 0 & \cdots & 0 \\
0 & 1 & 2 & 1 & 0 & 0 \\
& & \ddots & \ddots & \ddots & \\
0 & \cdots & 0 & 1 & 2 & 1 \\
(-)^{n} d_{2}^{2} / \xi_{n} & \cdots & 0 & 0 & 1 & \xi_{n-1} / \xi_{n}
\end{array}\right]
$$

where $\xi_{n}$ and the determinant are the same as for Matrix 1.

\subsection{KMS matrix: $\rho^{|i-j|}$}

The Kac-Murdock-Szegö matrix is the symmetric Toeplitz matrix $[13,18,33](\rho \neq 1, n>1)$ :

$$
A_{i j}=\rho^{|i-j|}, \quad i, j=1, \ldots, n
$$


It has the simple tridiagonal inverse

$$
A^{-1}=\frac{1}{1-\rho^{2}}\left[\begin{array}{ccccc}
1 & -\rho & 0 & \cdots & 0 \\
-\rho & 1+\rho^{2} & -\rho & \cdots & 0 \\
& \ddots & \ddots & \ddots & \\
0 & \cdots & -\rho & 1+\rho^{2} & -\rho \\
0 & \cdots & 0 & -\rho & 1
\end{array}\right] .
$$

The determinant is $|A|=\left(1-\rho^{2}\right)^{n-1}$. For a Matlab program which generates the LU decomposition of this matrix, see kms . m in [15], and for its eigenvalues see [13, p69]. This is the only form of a symmetric Toeplitz matrix whose inverse is a tridiagonal matrix; this can be shown by using the result that the inverse of a symmetric irreducible nonsingular tridiagonal matrix $T$ is of the general form $[2,4,5,23$, 24]

$$
T_{i j}^{-1}= \begin{cases}u_{i} v_{j}, & i \leq j \\ u_{j} v_{i}, & i>j\end{cases}
$$

The matrix $P$ of [34] is a KMS matrix, except it differs by the factor $\rho^{n-1}$, with $\rho=1 / q$. Given the matrix $T$ the vectors $u, v$ are easy to derive, see $\S 3.1$ or [8]; this and similar results are constantly being rediscovered $[7,17,35$, e.g.].

The KMS matrix and some of its generalizations given below are semiseparable matrices [10].

\subsection{Nonsymmetric KMS matrix}

The nonsymmetric version of the KMS matrix is:

$$
A_{i j}=\left\{\begin{array}{lll}
\rho^{j-i}, & i<j, \quad i, j=1, \ldots, n \\
\sigma^{i-j}, & i>j, \quad i, j=1, \ldots, n \\
1, & i=j .
\end{array}\right.
$$


Its inverse is

$$
A^{-1}=\frac{1}{1-\sigma \rho}\left[\begin{array}{ccccc}
1 & -\rho & 0 & \cdots & 0 \\
-\sigma & 1+\sigma \rho & -\rho & \cdots & 0 \\
& \ddots & \ddots & \ddots & \\
0 & \cdots & -\sigma & 1+\sigma \rho & -\rho \\
0 & \cdots & 0 & -\sigma & 1
\end{array}\right] .
$$

The determinant is $|A|=(1-\sigma \rho)^{n-1}$.

\subsection{Generalized KMS matrix: $\alpha+\beta \rho^{|i-j|}$}

A generalized symmetric KMS matrix is:

$$
A_{i j}= \begin{cases}\alpha+\beta \rho^{|i-j|}, & i, j=1, \ldots, n \\ \alpha+\beta, & i=j\end{cases}
$$

Its inverse is

$$
A^{-1}=\frac{1}{f_{n}\left(1-\rho^{2}\right)}\left[\begin{array}{rrrrrrr}
d_{0} & a_{0} & b & \cdots & b & b & c \\
a_{0} & d & a & e & \cdots & e & b \\
b & a & d & a & e & \cdots & b \\
& & & \ddots & \ddots & \ddots & \\
b & e & \cdots & e & a & d & a_{0} \\
c & b & b & \cdots & b & a_{0} & d_{0}
\end{array}\right] .
$$

where

$$
\begin{aligned}
d & =-1-\rho-\rho^{2}-\rho^{3}+\alpha\left\{-n_{1}+n_{5} \rho-n_{3} \rho^{2}(1-\rho)\right\} / \beta \\
a & =\rho(1+\rho)+\alpha\left(1+n_{3} \rho-n_{5} \rho^{2}-\rho^{3}\right) / \beta \\
a_{0} & =\rho(1+\rho)+\alpha\left(1+n_{2} \rho-n_{3} \rho^{2}\right) / \beta \\
d_{0} & =-1-\rho+\alpha\left(n_{3} \rho-n_{1}\right) / \beta
\end{aligned}
$$


$b=(1-\rho)^{2} \alpha / \beta, c=(1-\rho) \alpha / \beta, e=(1-\rho)^{3} \alpha / \beta, n_{m}=n-m$, $f_{n}=-n \alpha-\beta(1+\rho)+n_{2} \alpha \rho$. For example $(n=8, \alpha=1, \beta=2$, $\rho=2)$ :

$$
\begin{array}{r}
A^{-1}=\left[\begin{array}{rrrrrrrr}
3 & 5 & 9 & 17 & 33 & 65 & 129 & 257 \\
5 & 3 & 5 & 9 & 17 & 33 & 65 & 129 \\
9 & 5 & 3 & 5 & 9 & 17 & 33 & 65 \\
17 & 9 & 5 & 3 & 5 & 9 & 17 & 33 \\
33 & 17 & 9 & 5 & 3 & 5 & 9 & 17 \\
65 & 33 & 17 & 9 & 5 & 3 & 5 & 9 \\
129 & 65 & 33 & 17 & 9 & 5 & 3 & 5 \\
257 & 129 & 65 & 33 & 17 & 9 & 5 & 3
\end{array}\right]^{-1} \\
=-\frac{1}{12}\left[\begin{array}{rrrrrrrr}
3 & -5 & -1 & -1 & -1 & -1 & -1 & 1 \\
-5 & 11 & -3 & 1 & 1 & 1 & 1 & -1 \\
-1 & -3 & 11 & -3 & 1 & 1 & 1 & -1 \\
-1 & 1 & -3 & 11 & -3 & 1 & 1 & -1 \\
-1 & 1 & 1 & -3 & 11 & -3 & 1 & -1 \\
-1 & 1 & 1 & 1 & -3 & 11 & -3 & -1 \\
-1 & 1 & 1 & 1 & 1 & -3 & 11 & -5 \\
1 & -1 & -1 & -1 & -1 & -1 & -5 & 3
\end{array}\right]^{-1}
\end{array}
$$

We have quoted this matrix partly because its inverse, which although dense depends on only seven parameters, has a form which occurs in other cases. We quote four of these:

1. the centrosymmetric [3] Toeplitz matrix $(n>2, d \neq 0)$

$$
A_{i j}=c+d|i-j|+e(i-j)^{2}, \quad i, j=1, \ldots, n ;
$$

2. the matrix

$$
A_{i j}=c+d(-1)^{i-j}|i-j|, \quad i, j=1, \ldots, n
$$

which is apparently similar to (1) but whose inverse is dense; 
3. the matrix

$$
A_{i j}=c+(-1)^{i-j}\left\{d|i-j|+e(i-j)^{2}\right\}, \quad i, j=1, \ldots, n ;
$$

4. and finally the matrix

$$
A_{i j}=d+\alpha \sin (\rho|i-j|)+\beta \cos (\rho|i-j|), \quad i, j=1, \ldots, n .
$$

Because the inverse of these matrices has the form (3) it is not difficult to find explicit expressions for their inverses. We can also obtain expressions for the nonsymmetric case but they are somewhat more complex.

For another generalization of the KMS matrix, see [30].

\subsection{Hyperbolic matrix: $\alpha \rho^{-|i-j|}+\beta \rho^{|i-j|}$}

The symmetric Toeplitz matrix

$$
A_{i j}= \begin{cases}\alpha \rho^{-|i-j|}+\beta \rho^{|i-j|} & i, j=1, \ldots, n \\ \alpha+\beta & i=j\end{cases}
$$

has the inverse

$$
A^{-1}=\frac{1}{(\alpha-\beta)\left(\rho^{2}-1\right)}\left[\begin{array}{cccccc}
d_{0} & -\rho & 0 & \ldots & 0 & c \\
-\rho & 1+\rho^{2} & -\rho & 0 & \ldots & 0 \\
0 & -\rho & 1+\rho^{2} & -\rho & 0 & \ldots \\
& & \ddots & \ddots & \ddots & \\
0 & \ldots & 0 & -\rho & 1+\rho^{2} & -\rho \\
c & 0 & \cdots & 0 & -\rho & d_{0}
\end{array}\right]
$$

where $d_{0}=\rho^{2} f_{2 n-4} / f_{2 n-2}, c=\alpha \beta \rho^{n-1}\left(1-\rho^{2}\right) / f_{2 n-2}$ and $f_{k}=$ $\alpha^{2}-\beta^{2} \rho^{k}$. 
The determinant of $A$ is $|A|=(\alpha-\beta)^{n-2}\left(\rho^{2}-1\right)^{n-1} f_{2 n-2} / \rho^{2 n-2}$.

We now write $A$ in terms of hyperbolic functions, partly for reasons of comparison with the next section, but also because of the beauty of the matrix; we also generalize to a nonsymmetric matrix:

$$
A_{i j}= \begin{cases}\alpha \sinh (\rho|i-j|)+\beta \cosh (\rho|i-j|) & i \leq j, i, j=1, \ldots, n \\ \gamma \sinh (\rho|i-j|)+\beta \cosh (\rho|i-j|) & i \geq j, i, j=1, \ldots, n\end{cases}
$$

which has the inverse

$$
A^{-1}=\frac{1}{\alpha+\gamma}\left[\begin{array}{cccccc}
d_{0} & a & 0 & \cdots & 0 & f \\
a & d & a & 0 & \cdots & 0 \\
0 & a & d & a & \cdots & 0 \\
& & \ddots & \ddots & \ddots & \\
0 & \cdots & 0 & a & d & a \\
e & 0 & \cdots & 0 & a & d_{0}
\end{array}\right]
$$

where

$$
\begin{aligned}
a & =\operatorname{csch} \rho \\
d & =-2 \operatorname{coth} \rho \\
e & =-\left(\beta g_{n-2} \operatorname{csch} \rho+g_{n-1}(\gamma-\beta \operatorname{coth} \rho) / D\right. \\
f & =-\left(\beta h_{n-2} \operatorname{csch} \rho+h_{n-1}(\alpha-\beta \operatorname{coth} \rho) / D\right. \\
d_{0} & =\left(g_{n-2} h_{n-1} \operatorname{csch} \rho+\beta(\gamma-\beta \operatorname{coth} \rho)\right) / D \\
D & =\beta^{2}-g_{n-1} h_{n-1} \\
h_{k} & =\alpha \sinh (\rho k)+\beta \cosh (\rho k) \\
g_{k} & =\gamma \sinh (\rho k)+\beta \cosh (\rho k)
\end{aligned}
$$

As we shall see this has a perfect analogue in the trigonometric functions. 


\subsection{Trigonometric matrix:}

$$
\alpha \sin (\rho|i-j|)+\beta \cos (\rho|i-j|)
$$

The previous matrix leads to our next matrix: the nonsymmetric trigonometric Toeplitz matrix

$$
A_{i j}= \begin{cases}\alpha \sin (\rho|i-j|)+\beta \cos (\rho|i-j|) & i \leq j, i, j=1, \ldots, n \\ \gamma \sin (\rho|i-j|)+\beta \cos (\rho|i-j|) & i \geq j, i, j=1, \ldots, n\end{cases}
$$

which has the inverse

$$
A^{-1}=\frac{1}{\alpha+\gamma}\left[\begin{array}{cccccc}
d_{0} & a & 0 & \cdots & 0 & f \\
a & d & a & 0 & \cdots & 0 \\
0 & a & d & a & \cdots & 0 \\
& & \ddots & \ddots & \ddots & \\
0 & \cdots & 0 & a & d & a \\
e & 0 & \cdots & 0 & a & d_{0}
\end{array}\right]
$$

where

$$
\begin{aligned}
a & =\csc \rho \\
d & =-2 \cot \rho \\
e & =-\left(\beta g_{n-2} \csc \rho+g_{n-1}(\gamma-\beta \cot \rho) / D\right. \\
f & =-\left(\beta h_{n-2} \csc \rho+h_{n-1}(\alpha-\beta \cot \rho) / D\right. \\
d_{0} & =\left(g_{n-2} h_{n-1} \csc \rho+\beta(\gamma-\beta \cot \rho)\right) / D \\
D & =\beta^{2}-g_{n-1} h_{n-1} \\
h_{k} & =\alpha \sin (\rho k)+\beta \cos (\rho k) \\
g_{k} & =\gamma \sin (\rho k)+\beta \cos (\rho k)
\end{aligned}
$$

The curious thing about this matrix is that the asymmetry is confined to the corner elements $e$ and $f$. We will quote a (symmetric) 
example: let $n=8, \alpha=\beta=\gamma=1, \rho=\pi / 4$ then

$$
\begin{aligned}
A^{-1}= & {\left[\begin{array}{cccccccc}
1 & \sqrt{2} & 1 & 0 & -1 & -\sqrt{2} & -1 & 0 \\
\sqrt{2} & 1 & \sqrt{2} & 1 & 0 & -1 & -\sqrt{2} & -1 \\
1 & \sqrt{2} & 1 & \sqrt{2} & 1 & 0 & -1 & -\sqrt{2} \\
0 & 1 & \sqrt{2} & 1 & \sqrt{2} & 1 & 0 & -1 \\
-1 & 0 & 1 & \sqrt{2} & 1 & \sqrt{2} & 1 & 0 \\
-\sqrt{2} & -1 & 0 & 1 & \sqrt{2} & 1 & \sqrt{2} & 1 \\
-1 & -\sqrt{2} & -1 & 0 & 1 & \sqrt{2} & 1 & \sqrt{2} \\
0 & -1 & -\sqrt{2} & -1 & 0 & 1 & \sqrt{2} & 1
\end{array}\right] } \\
= & \frac{1}{\sqrt{2}}\left[\begin{array}{ccccccccc}
0 & 1 & 0 & 0 & 0 & 0 & 0 & 1 \\
1 & -\sqrt{2} & 1 & 0 & 0 & 0 & 0 & 0 \\
0 & 1 & -\sqrt{2} & 1 & 0 & 0 & 0 & 0 \\
0 & 0 & 1 & -\sqrt{2} & 1 & 0 & 0 & 0 \\
0 & 0 & 0 & 1 & -\sqrt{2} & 1 & 0 & 0 \\
0 & 0 & 0 & 0 & 1 & -\sqrt{2} & 1 & 0 \\
0 & 0 & 0 & 0 & 0 & 1 & -\sqrt{2} & 1 \\
1 & 0 & 0 & 0 & 0 & 0 & 1 & 0
\end{array}\right]
\end{aligned}
$$

\section{$2 \quad$ Fiedler's matrix: $c_{j}-c_{i}$}

In a surprising result, Fiedler [28], stated that the inverse of

$$
C_{i j}=\left\{\begin{aligned}
c_{j}-c_{i}, & i<j, \quad i, j=1, \ldots, n \\
c_{i}-c_{j}, & i>j, \quad i, j=1, \ldots, n \\
0, & i=j,
\end{aligned}\right.
$$

$(n>2)$ is also given by a tridiagonal matrix except for $c_{1 n}^{-1}$ and $c_{n 1}^{-1} \neq$ 0: Put $d_{1}=1 /\left(c_{1}-c_{2}\right)-1 /\left(c_{1}-c_{n}\right), d_{i}=1 /\left(c_{i-1}-c_{i}\right)+1 /\left(c_{i}-c_{i+1}\right)$, 


$$
\begin{gathered}
i=2, \ldots, n-1, d_{n}=1 /\left(c_{n-1}-c_{n}\right)-1 /\left(c_{1}-c_{n}\right) \text {, then } \\
C_{i j}^{-1}=\frac{1}{2}\left[\begin{array}{cccccc}
d_{1} & \frac{1}{c_{2}-c_{1}} & 0 & \ldots & 0 & \frac{1}{c_{n}-c_{1}} \\
\frac{1}{c_{2}-c_{1}} & d_{2} & \frac{1}{c_{3}-c_{2}} & 0 & \ldots & 0 \\
& \ddots & \ddots & \ddots & & \\
& & \ddots & \ddots & \ddots & \\
0 & \ldots & 0 & \frac{1}{c_{n-1}-c_{n-2}} & \frac{d_{n-1}}{c_{n}-c_{n-1}} \\
\frac{1}{c_{n}-c_{1}} & 0 & \ldots & 0 & \frac{1}{c_{n}-c_{n-1}} & d_{n}
\end{array}\right]
\end{gathered}
$$

We have printed this in full as there was a slight error in the original publication. Fiedler also gave an expression for the determinant of this matrix: $|C|=-(-1)^{n} 2^{n-2} \prod_{j=1}^{n-1}\left(c_{j+1}-c_{j}\right)\left(c_{n}-c_{1}\right),(n>1)$.

\subsection{Generalized nonsymmetric Fiedler's matrix: $d+p c_{i}+q c_{j}$}

We have generalized Fiedler's matrix to:

$$
C_{i j}=\left\{\begin{array}{lll}
d+p c_{i}+q c_{j}, & i<j, & i, j=1, \ldots, n \\
d+r c_{i}+s c_{j}, & i>j, & i, j=1, \ldots, n \\
d+(p+q) c_{i}, & i=j,
\end{array}\right.
$$

where we require $r+s=p+q$ to make the upper and lower triangles consistent. The inverse is very similar to Fiedler's matrix. Put

$$
\begin{aligned}
e & =s r / \xi_{1 n} \\
f & =p q / \xi_{1 n} \\
d_{1} & =\frac{\xi_{2 n}}{\left(c_{1}-c_{2}\right) \xi_{1 n}} \\
d_{n} & =\frac{\xi_{1 n-1}}{\left(c_{n-1}-c_{n}\right) \xi_{1 n}} \\
\xi_{i j} & =d(p-r)+p s c_{i}-q r c_{j}
\end{aligned}
$$


otherwise $d_{i}$ is as above, then provided $C^{-1}$ exists, for $n>2$ it is given by

$$
C_{i j}^{-1}=\frac{1}{r-p}\left[\begin{array}{ccccc}
d_{1} & \frac{1}{c_{2}-c_{1}} & \ldots & 0 & f \\
\frac{1}{c_{2}-c_{1}} & d_{2} & \frac{1}{c_{3}-c_{2}} & \cdots & 0 \\
& \ddots & \ddots & \ddots & \\
0 & \cdots & \frac{1}{c_{n-1}-c_{n-2}} & \frac{d_{n-1}}{c_{n}-c_{n-1}} \\
e & 0 & \cdots & \frac{1}{c_{n}-c_{n-1}} & d_{n}
\end{array}\right]
$$

Example: $n=8, d=2, p=1, q=1, r=4$, and $c=\left\{\begin{array}{llllllll}1 & 2 & 0 & 1 & 2 & 0 & 1 & 2\end{array}\right\}:$

$$
\begin{aligned}
A^{-1}= & {\left[\begin{array}{rrrrrrrr}
4 & 5 & 3 & 4 & 5 & 3 & 4 & 5 \\
8 & 6 & 4 & 5 & 6 & 4 & 5 & 6 \\
0 & -2 & 2 & 3 & 4 & 2 & 3 & 4 \\
4 & 2 & 6 & 4 & 5 & 3 & 4 & 5 \\
8 & 6 & 10 & 8 & 6 & 4 & 5 & 6 \\
0 & -2 & 2 & 0 & -2 & 2 & 3 & 4 \\
4 & 2 & 6 & 4 & 2 & 6 & 4 & 5 \\
8 & 6 & 10 & 8 & 6 & 10 & 8 & 6
\end{array}\right] } \\
= & \frac{1}{6}\left[\begin{array}{rrrrrrrrr}
-2 \frac{1}{4} & 2 & 0 & 0 & 0 & 0 & 0 & -\frac{1}{8} \\
2 & -1 & -1 & 0 & 0 & 0 & 0 & 0 \\
0 & -1 & -1 & 2 & 0 & 0 & 0 & 0 \\
0 & 0 & 2 & -4 & 2 & 0 & 0 & 0 \\
0 & 0 & 0 & 2 & -1 & -1 & 0 & 0 \\
0 & 0 & 0 & 0 & -1 & -1 & 2 & 0 \\
0 & 0 & 0 & 0 & 0 & 2 & -4 & 2 \\
1 & 0 & 0 & 0 & 0 & 0 & 2 & -1 \frac{1}{2}
\end{array}\right]
\end{aligned}
$$

The only further generalization of this matrix which still has a sparse inverse that we have found at this stage is to add the rank one term: $+\rho c_{i} c_{j}$ to $C$. The matrices of $\S 1.1$ and $\S 1.2$ are instances of a Fiedler matrix. 


\section{Inverse of band Toeplitz matrix}

Rozsa [24] gave formulae for the inverses of band matrices; we will derive equivalent results in a different and more direct way; note that our definition of $p$ and $q$ is different to Rozsa. In his treatment, he has to evaluate $4(p+q)$ determinants of order $p+q$, whereas here we need to solve two systems of order $p+q$. The terms in Rozsa's expression for the inverse are more concise, being sums of rank $p$ or $q$ (which agrees with Barrett [6]), whereas here we derive sums of $p+q$ terms.

Consider the band Toeplitz matrix

$$
A=\left[\begin{array}{cccccc}
c_{0} & c_{1} & \cdots & c_{q} & 0 & 0 \\
c_{-1} & c_{0} & c_{1} & \cdots & c_{q} & 0 \\
& \ddots & \ddots & \ddots & & \\
& & \ddots & \ddots & \ddots & \\
0 & \cdots & c_{-p} & \cdots & c_{0} & c_{1} \\
0 & 0 & \cdots & c_{-p} & \cdots & c_{0}
\end{array}\right]
$$

where $p \geq 0, q \geq 0, p+q \geq 1$. That is

$$
A_{i j}= \begin{cases}c_{j-i}, & -p \leq j-i \leq q \\ 0, & \text { otherwise }\end{cases}
$$

We invert this matrix using solutions to the difference equation (4).

Theorem 1 1. Let $r_{k}(i), k=1, \ldots, p+q$ be a set of $p+q$ linearly independent solutions to the difference equation

$$
c_{-p} r(i-p)+c_{1-p} r(i-p+1)+\cdots+c_{q} r(i+q)=0,
$$

for $i=\ldots, 0,1,2, \ldots$ 
2. Construct two solutions to (4):

$$
\begin{gathered}
P_{i j}=\sum_{k=1}^{p+q} a_{k}(j) r_{k}(i) ; \\
Q_{i j}=\sum_{k=1}^{p+q}\left[a_{k}(j)-z_{k}(j)\right] r_{k}(i) .
\end{gathered}
$$

3. To find $a_{k}$ and $z_{k}$ solve:

$$
\begin{gathered}
P_{i j}=0, \quad i=1-p, \ldots, 0, \quad \text { if } p>0 ; \\
Q_{i j}=0, \quad i=n, \ldots, n+q, \quad \text { if } q>0 ; \\
P_{i j}=Q_{i j}, \quad i=j-p+1, \ldots, j+q-1, \\
\quad \text { if } p+q>1 ; \\
c_{-p} A_{j-p, j}^{-1}+c_{1-p} A_{j-p+1, j}^{-1}+\cdots+c_{q} A_{j+q, j}^{-1}=1 .
\end{gathered}
$$

Since $P$ satisfies (4) the last condition becomes

$$
-P_{j+q, j}+Q_{j+q, j}=1 / c_{q}, \quad \text { if } p+q>1 .
$$

We assert that the inverse of $A$ is

$$
A_{i j}^{-1}= \begin{cases}P_{i j}, & 1 \leq i \leq j+q-1 \\ Q_{i j}, & j-p+1 \leq i \leq n\end{cases}
$$

Proof: Because $P, Q$ as functions of $i$ satisfy the difference equation (4) it is clear that $B_{i j}=\sum A_{i k} P_{k j}$ (or $A_{i k} Q_{k j}$ ) will be zero in general except perhaps for $i$ near 1 or $n$. Equations (6) and (7) ensure that $B_{i j}=0$ for $i<p+1$ or $i>n-q$. Next, (10) ensures that $B_{i i}=1$, and finally (8) ensures that $P$ and $Q$ are consistent over their common domain. Hence $B=I$ and we have constructed the inverse of $A$. 
Because of the way we chose $a_{k}$ and $z_{k}$, the system (6-10) of $2(p+q)$ equations for $a_{k}(j)$ and $z_{k}(j)$ separates into two systems of size $p+q$.

To solve these, first we solve equations (8) and (10)

$$
\sum_{k=1}^{p+q} r_{k}(j-p+l) z_{k}(j)= \begin{cases}0, & l=1, \ldots, p+q-1 \\ -1 / c_{q}, & l=p+q\end{cases}
$$

These will always be solvable as the $r_{k}$ are linearly independent. Then we solve the $p+q$ equations

$$
\sum_{k=1}^{p+q} r_{k}(l) a_{k}(j)= \begin{cases}0, & l=1-p, \ldots, 0 \\ \sum_{k=1}^{p+q} r_{k}(l) z_{k}(j), & l=n+1, \ldots, n+q\end{cases}
$$

These equations should be solved symbolically, giving explicit expressions for $a_{k}(j)$ and $z_{k}(j)$ and hence $P$ and $Q$, in terms of $i$ and $j$.

We also note that if $A$ is singular then the determinant

$$
\left|r_{k}(l)\right|=0
$$

where $l=1-p, \ldots, 0, n+1, \ldots, n+q$. The reverse of this is not necessarily true, thus (14) can have parasitic solutions, which correspond to the occurrence of equal roots of (4). Thus we can use (14) to obtain the eigenvalues of $A$, if we replace $c_{0}$ by $c_{0}-$ $\lambda$. We do not need to solve (4) directly; rather we use symmetric functions of the roots of (4) to simplify (14), for example if (4) has the solutions $r(i)=r_{k}^{i}$ then we have $\prod r_{k}=(-)^{p+q} c_{-p} / c_{q}$. We will not explore this technique further, as other methods [19, 25] are more direct. 


\subsection{Example: General tridiagonal matrix}

Consider the tridiagonal matrix

$$
A=\left[\begin{array}{rrrrrr}
c_{0} & c_{1} & 0 & \ldots & 0 & 0 \\
c_{-1} & c_{0} & c_{1} & 0 & \ldots & 0 \\
& \ddots & \ddots & \ddots & & \\
& & \ddots & \ddots & \ddots & \\
0 & \ldots & 0 & c_{-1} & c_{0} & c_{1} \\
0 & 0 & \ldots & 0 & c_{-1} & c_{0}
\end{array}\right] .
$$

Many authors have inverted particular cases of this matrix [20], but for the convenience of the reader we quote the general solution. To find the inverse of $A$ we first solve (4) in the usual way, putting $r(i)=r^{i}$, giving $c_{-1}+r c_{0}+r^{2} c_{1}=0$. We need to consider three cases: two unequal real roots, two equal roots or two complex roots. We have $p=q=1$. First we solve (12) giving

$$
\begin{aligned}
& z_{1}(j)=-r_{2}(j) / c_{1} D(j), \\
& z_{2}(j)=r_{1}(j) / c_{1} D(j),
\end{aligned}
$$

where

$$
D(j)=r_{1}(j+1) r_{2}(j)-r_{1}(j) r_{2}(j+1) .
$$

We then solve (13) for the three cases, the inverse being given by (11).

Unequal real roots The roots are $r_{1}, r_{2}=\left(-c_{0} \pm \sqrt{c_{0}^{2}-4 c_{-1} c_{1}}\right) / 2 c_{1}$, and $r_{k}(i)=r_{k}^{i}, r_{1} \neq r_{2}$. Then

$$
P_{i j}=-\frac{\left(r_{1}^{i}-r_{2}^{i}\right)\left(r_{1}^{n+1-j}-r_{2}^{n+1-j}\right)}{c_{1}\left(r_{1}-r_{2}\right)\left(r_{1}^{n+1}-r_{2}^{n+1}\right)}
$$




$$
Q_{i j}=\frac{\left(r_{1}^{-j}-r_{2}^{-j}\right)\left(r_{1}^{n+1} r_{2}^{i}-r_{2}^{n+1} r_{1}^{i}\right)}{c_{1}\left(r_{1}-r_{2}\right)\left(r_{1}^{n+1}-r_{2}^{n+1}\right)} .
$$

We also record the latter in a form emphasising the symmetry:

$$
Q_{i j}=-\frac{\left(r_{1}^{j}-r_{2}^{j}\right)\left(r_{1}^{n+1-i}-r_{2}^{n+1-i}\right)}{c_{1}\left(r_{1}-r_{2}\right)\left(r_{1}^{n+1}-r_{2}^{n+1}\right)}\left(\frac{c_{-1}}{c_{1}}\right)^{i-j} .
$$

Haley [14] gave these in terms of hyperbolic functions, and we quote them here for completeness. We assume without loss of generality that $c_{0}>0$. Put $\cosh \theta=c_{0} / 2 \sqrt{c_{-1} c_{1}}$, $r=-\sqrt{c_{-1} / c_{1}}$ if $c_{1}>0$ and $r=\sqrt{c_{-1} / c_{1}}$ if $c_{1}<0$. Then

$$
\begin{aligned}
P_{i j} & =\frac{r^{i-j} \sinh i \theta \sinh (n+1-j) \theta}{\sqrt{c_{-1} c_{1}} \sinh \theta \sinh (n+1) \theta}, \\
Q_{i j} & =\frac{r^{i-j} \sinh j \theta \sinh (n+1-i) \theta}{\sqrt{c_{-1} c_{1}} \sinh \theta \sinh (n+1) \theta} .
\end{aligned}
$$

These formulae still apply if $c_{-1} c_{1}<0$ but because of the imaginary terms, (15) is to be preferred.

Equal roots Here $r_{1}(i)=r^{i}, r_{2}(i)=i r^{i}, r=-c_{0} / 2 c_{1}$. Then

$$
\begin{gathered}
P_{i j}=-\frac{2 r^{i-j} i(j-n-1)}{c_{0}(n+1)}, \\
Q_{i j}=-\frac{2 r^{i-j} j(i-n-1)}{c_{0}(n+1)} .
\end{gathered}
$$

Complex roots Here $r_{1}(i)=r^{i} \cos i \theta, r_{2}(i)=r^{i} \sin i \theta$, where $r=$ $\sqrt{c_{-1} / c_{1}}, \cos \theta=-c_{0} / 2 r c_{1}$. Then

$$
P_{i j}=-\frac{r^{i-j} \sin i \theta \sin (n+1-j) \theta}{\sqrt{c_{-1} c_{1}} \sin \theta \sin (n+1) \theta},
$$




$$
Q_{i j}=-\frac{r^{i-j} \sin j \theta \sin (n+1-i) \theta}{\sqrt{c_{-1} c_{1}} \sin \theta \sin (n+1) \theta} .
$$

If $c_{1}<0$, the signs of $P$ and $Q$ should be changed.

As our objective was to give explicit expressions for the inverse, we will show how to avoid evaluating $\theta$ and the trigonometric functions above. The Chebyshev polynomials [1] of the first and second kinds are $T_{n}(x)=\cos n \theta, U_{n}(x)=\sin (n+1) \theta / \sin \theta$, where $x=\cos \theta$; both satisfy the recurrence $T_{n}=2 x T_{n-1}-T_{n-2}$, the first few terms being $T_{0}=1, T_{1}(x)=x, U_{0}=1, U_{1}=2 x$. Putting $x=\cos \theta=$ $-c_{0} / 2 r c_{1},(17)$ becomes

$$
\begin{aligned}
& P_{i j}=-r^{i-j} U_{i-1}(x)\left[T_{j}(x)-U_{j-1}(x) T_{n+1}(x) / U_{n}(x)\right] / \sqrt{c_{-1} c_{1}}, \\
& Q_{i j}=-r^{i-j} U_{j-1}(x)\left[T_{i}(x)-U_{i-1}(x) T_{n+1}(x) / U_{n}(x)\right] / \sqrt{c_{-1} c_{1}} .
\end{aligned}
$$

Note in passing that these forms of the inverse agree with Barrett's theorem [6] that $A$ is tridiagonal iff $A^{-1}$ is of the form

$$
A_{i, j}^{-1}= \begin{cases}x_{i} y_{j}, & i \leq j \\ u_{i} v_{j}, & i>j\end{cases}
$$

provided $u_{i} v_{i}=x_{i} y_{i}, i=1, \ldots, n$. 


\subsection{Example: third order difference operator matrix}

Consider the nonsymmetric third order difference operator matrix $(p=2, q=1)$

$$
A=\left[\begin{array}{cccccc}
3 & -1 & 0 & 0 & \cdots & 0 \\
-3 & 3 & -1 & 0 & \cdots & 0 \\
1 & -3 & 3 & -1 & & 0 \\
& \ddots & \ddots & \ddots & \ddots & \\
0 & & 1 & -3 & 3 & -1 \\
0 & \cdots & 0 & 1 & -3 & 3
\end{array}\right]
$$

The inverse of this matrix is

$$
A_{i j}^{-1}= \begin{cases}a_{0}(j) i(i+1), & i \leq j \\ b_{0}(j) i^{2}+b_{1}(j) i+b_{2}(j), & i \geq j-1\end{cases}
$$

where

$$
\begin{aligned}
& a_{0}(j)=(1-j+n)(2-j+n) / c, \\
& b_{0}(j)=j(-3+j-2 n) / c \\
& b_{1}(j)=j\left(1+j+4 n+2 n^{2}\right) / c, \\
& b_{2}(j)=-(-1+j) j / 2, \\
& c \quad=2(n+1)(n+2) .
\end{aligned}
$$

In this case $P$ and $Q$ are second-order polynomials in $i$ and $j$, which are equal for $i=j-1, j$.

For example, the first column of $A^{-1}$ is $Q_{i 1}=i(n+1-i) /(n+2)$.

Theorem 2 For $n \geq 1$, the determinant of the third order difference operator matrix $A_{n}$ is

$$
\left|A_{n}\right|=(n+1)(n+2) / 2 .
$$


Proof: Partition $A_{n}$ as

$$
A_{n+1}=\left[\begin{array}{cc}
B & C \\
D & A_{n}
\end{array}\right]
$$

where $B=3, D^{\prime}=\left[\begin{array}{lllll}-3 & 1 & 0 & 0 & \cdots\end{array}\right]$ and $C=\left[\begin{array}{llll}-1 & 0 & 0 & \cdots\end{array}\right]$. Then using the well known result $\left|A_{n+1}\right|=\left|A_{n}\right|\left|B-C A_{n}^{-1} D\right|$ and the above expression for the inverse, we get

$$
C A_{n}^{-1} D=2 n /(n+1) .
$$

The proof then follows by induction.

\subsection{Example: fourth order difference operator matrix}

Consider the symmetric Toeplitz matrix $(p=q=2)$

$$
A=\left[\begin{array}{ccccccc}
6 & -4 & 1 & 0 & \cdots & 0 & 0 \\
-4 & 6 & -4 & 1 & 0 & \cdots & 0 \\
1 & -4 & 6 & -4 & 1 & & 0 \\
& \ddots & \ddots & \ddots & \ddots & \ddots & \\
& & \ddots & \ddots & \ddots & \ddots & \\
0 & \cdots & 0 & 1 & -4 & 6 & -4 \\
0 & 0 & \cdots & 0 & 1 & -4 & 6
\end{array}\right]
$$

This matrix was also considered in [2] and [16] using less general methods. We can write down the first column of the inverse of this matrix by inspection: since the solutions to the difference equation (4) in this case are $r(i)=\left\{1, i, i^{2}, i^{3}\right\}$ and it follows from (7) that $Q_{i 1}=0$ for $i=0, n+1$ and $n+2$ we have

$$
Q_{i 1}=c i(n+1-i)(n+2-i)
$$


and $c$ can be found from $6 Q_{11}-4 Q_{21}+Q_{31}=1$ giving

$$
A_{i, 1}^{-1}=i \frac{(n+1-i)(n+2-i)}{(n+2)(n+3)} .
$$

Note that since $A$ is centrosymmetric, having found the first column, we then have the other first and last columns and rows, and we can then use the formula of Trench [32, p207], [11, 31, p188], namely if $T_{n}$ is a Toeplitz matrix, then putting $T_{n}^{-1}=\left(h_{i j}\right)$, provided $h_{00} \neq 0$ we have

$$
h_{i j}=h_{i-1, j-1}+\left(h_{00}\right)^{-1}\left[h_{i 0} h_{0 j}-h_{n-j+1,0} h_{0, n-i+1}\right], \quad 0 \leq i, j \leq n .
$$

In full, the inverse of the fourth-order difference operator matrix (18) is

$$
A_{i j}^{-1}= \begin{cases}a_{0} i^{3}+a_{1} i^{2}+a_{2} i, & i \leq j+1 \\ b_{0} i^{3}+b_{1} i^{2}+b_{2} i+b_{3}, & i \geq j-1\end{cases}
$$

where we have dropped the $j$ subscript to improve readability, and

$$
\begin{aligned}
& a_{0}=-(3+2 j+n) d_{j} / c \\
& a_{1}=3 j(1+n) d_{j} / c \\
& a_{2}=(3+5 j+n+3 j n) d_{j} / c \\
& b_{0}=(5-2 j+3 n) e_{j} / c \\
& b_{1}=-3(1+n)(4-j+2 n) e_{j} / c \\
& b_{2}=\left(1+5 j+12 n+3 j n+12 n^{2}+3 n^{3}\right) e_{j} / c \\
& b_{3}=(1-j) e_{j} / 6
\end{aligned}
$$

and $d_{j}=(n-j+1)(n-j+2), e_{j}=j(j+1), c=6(n+1)(n+2)(n+3)$.

Here $P$ and $Q$ are cubic polynomials in $i$ and $j$, which are equal for $i=j-1, j, j+1$. The inverse is of course symmetric, so we do not have to derive $b_{k}$ in this case. 
Theorem 3 For $n \geq 1$, the determinant of the fourth order difference operator matrix $A_{n}$ is

$$
\left|A_{n}\right|=(n+1)(n+2)^{2}(n+3) / 12 .
$$

Proof: Partition $A_{n}$ as

$$
A_{n}=\left[\begin{array}{cc}
B & D^{\prime} \\
D & A_{n-1}
\end{array}\right]
$$

where $B=6, \quad D^{\prime}=\left[\begin{array}{lllll}-4 & 1 & 0 & 0 & \cdots\end{array}\right]$. Then using the well known result $\left|A_{n}\right|=\left|A_{n-1}\right|\left|B-D^{\prime} A_{n-1}^{-1} D\right|$ and the above expression for the inverse, we get

$$
D^{\prime} A_{n-1}^{-1} D=(5 n+6)(n-1) /(n(n+1)) .
$$

The proof then follows by induction.

\section{Tridiagonal matrix with modified corner elements}

Because many of the above examples had an inverse which was a tridiagonal matrix with constant diagonals except for the corner elements, we will derive the inverse of a matrix of this form.

The method of $\S 3$ extends easily to this case, which amounts to a change in the boundary conditions, and we can do the same for any first and last row, but our ansatz (5) will not apply to all $A^{-1}$ if we alter any other rows. 
Let

$$
A=\left[\begin{array}{rrrrrr}
d & c_{1} & 0 & \ldots & 0 & e \\
c_{-1} & c_{0} & c_{1} & 0 & \ldots & 0 \\
& \ddots & \ddots & \ddots & & \\
& & \ddots & \ddots & \ddots & \\
0 & \ldots & 0 & c_{-1} & c_{0} & c_{1} \\
e & 0 & \ldots & 0 & c_{-1} & d
\end{array}\right]
$$

In the above notation we have $p=q=1$, and $r_{k}(i)$ are two independent solutions of the difference equation $c_{-1} r(i)+c_{0} r(i+$ 1) $+c_{1} r(i+2)=0$. The inverse is

$$
A_{i j}^{-1}= \begin{cases}P_{i j}, & 1 \leq i \leq j \\ Q_{i j}, & j \leq i \leq n\end{cases}
$$

where $P, Q$ are as in (5). Proceeding as above we multiply rows $i=$ $1, j, n$ of $A$ by column $j$ of $A^{-1}$ to get the equations

$$
\begin{aligned}
d P_{1, j}+c_{1} P_{2 j}+e Q_{n j} & =0, \\
-P_{j+1, j}+Q_{j+1, j} & =1 / c_{1}, \\
e P_{1 j}+c_{-1} Q_{n-1, j}+d Q_{n j} & =0, \\
P_{j j} & =Q_{j j} .
\end{aligned}
$$

From $(20,22)$ we have again

$$
\begin{aligned}
& z_{1}(j)=-r_{2}(j) / c_{1} D(j), \\
& z_{2}(j)=r_{1}(j) / c_{1} D(j),
\end{aligned}
$$

as in $(15)$, and $(19,21)$ give

$$
\begin{aligned}
& f_{1} a_{1}(j)+f_{2} a_{2}(j)=e \sum_{k}^{2} z_{k}(j) r_{k}(n) \\
& g_{1} a_{1}(j)+g_{2} a_{2}(j)=\sum_{k}^{2} z_{k}(j)\left(c_{-1} r_{k}(n-1)+d r_{k}(n)\right),
\end{aligned}
$$


where

$$
\begin{gathered}
f_{k}=d r_{k}(1)+c_{1} r_{k}(2)+e r_{k}(n), \\
g_{k}=e r_{k}(1)+c_{-1} r_{k}(n-1)+d r_{k}(n) .
\end{gathered}
$$

Solving (23) gives $a_{k}$ and we apply (11) to give the inverse. It is not immediately obvious that (19-22) apply for $j=1$ and $j=n$; however, by working out these cases separately, they can be shown to conform to the above.

Example: $d=e=1, c=\{-2,3,-1\}$ we get $r(i)=\left\{1,2^{i}\right\}$ then

$$
A_{i j}^{-1}= \begin{cases}2^{i-1}-2^{n-1}+2^{n-j}, & i \leq j \\ 2^{i-1}-2^{n-1}+2^{n-j}+1-2^{i-j}, & i \geq j\end{cases}
$$

Comparing this to (15) we see that the inverse still consists of linear combinations and products of $2^{i}$ and $2^{-j}$. For $n=8$ this is

$$
\begin{aligned}
A^{-1}= & {\left[\begin{array}{rrrrrrrr}
1 & -1 & 0 & 0 & 0 & 0 & 0 & 1 \\
-2 & 3 & -1 & 0 & 0 & 0 & 0 & 0 \\
0 & -2 & 3 & -1 & 0 & 0 & 0 & 0 \\
0 & 0 & -2 & 3 & -1 & 0 & 0 & 0 \\
0 & 0 & 0 & -2 & 3 & -1 & 0 & 0 \\
0 & 0 & 0 & 0 & -2 & 3 & -1 & 0 \\
0 & 0 & 0 & 0 & 0 & -2 & 3 & -1 \\
1 & 0 & 0 & 0 & 0 & 0 & -2 & 1
\end{array}\right]^{-1} } \\
= & {\left[\begin{array}{rrrrrrrr}
1 & -63 & -95 & -111 & -119 & -123 & -125 & -126 \\
1 & -62 & -94 & -110 & -118 & -122 & -124 & -125 \\
1 & -61 & -92 & -108 & -116 & -120 & -122 & -123 \\
1 & -59 & -89 & -104 & -112 & -116 & -118 & -119 \\
1 & -55 & -83 & -97 & -104 & -108 & -110 & -111 \\
1 & -47 & -71 & -83 & -89 & -92 & -94 & -95 \\
1 & -31 & -47 & -55 & -59 & -61 & -62 & -63 \\
1 & 1 & 1 & 1 & 1 & 1 & 1 & 1
\end{array}\right] }
\end{aligned}
$$




\section{Conclusion}

We have described fifteen matrices and six examples, some of which are new, and all of which have easily constructible inverses. The hyperbolic, trigonometric and generalized Fiedler matrices are particularly striking. We described a simple method for inverting band Toeplitz matrices, which is extendable to other cases. We gave results for the tridiagonal case, in the hope that these were complete, easily applicable and useful, as whereas many authors quote particular cases, the complete treatment for all cases of the diagonals does not seem to be readily available. One form that recurs is the tridiagonal with modified corner elements, which we inverted.

At the time of publishing, FORTRAN programs which implement the formulae in this paper are available from http://anusf.anu. edu. au/ mld900/math/toeplitz/.

\section{References}

[1] M. Abramowitz and I. Stegun, Handbook of mathematical functions, (Dover, 1968), §22. E204

[2] E. L. Allgower, Exact inverses of certain band matrices, Numer. Math., 21, (1973), 279-284. E187, E190, E206

[3] A. L. Andrew, Centrosymmetric matrices, SIAM Rev., 40, (1998), 697-698. E192

[4] E. Asplund, Inverses of matrices $\left\{a_{i j}\right\}$ which satisfy $a_{i j}=0$ for $j>i+p$, Math. Scand. 7, (1959), 57-60. E190

[5] W. W. Barrett, A theorem on inverses of tridiagonal matrices, Linear Algebra Appl., 27, (1979), 211-217. E190 
[6] W. W. Barrett and P. J. Feinsilver, Inverses of banded matrices, Linear Algebra Appl., 41, (1981), 111-130. E199, E204

[7] S. S. Cheng and L. Y. Hsieh, Inverses of matrices arising from difference operators, Utilitas Mathematica, 38, (1990), 65-77. E187, E190

[8] P. Concus, G. H. Golub and G. Meurant, Block preconditioning for the conjugate gradient method, SIAM J. Sci. Stat. Comput., 6, (1985), 220-252. E187, E190

[9] M. L. Dow, Sparse inverse and characteristic polynomial of generalized arrow matrix, J. Austral. Math. Soc. B (E), 39, 667-677. E188

[10] I. Gohberg, T. Kailath and I. Koltracht, Linear complexity algorithms for semiseparable matrices, Integral Equations Operator Theory, 8, (1985), 780-804. E190

[11] G. H. Golub and C. F. Van Loan, Matrix Computations, Second ed., (The Johns Hopkins University Press, Baltimore, MD, 1989). E207

[12] R. T. Gregory and D. L. Karney, A Collection of Matrices for Testing Computational Algorithms, (Wiley Interscience, New York, 1969). E188

[13] U. Grenander and G. Szegö, Toeplitz forms and their applications (Uni. Calif. Press, 1958) E186, E189, E190

[14] S. B. Haley, Solution of band matrix equations by projection-recurrence, Linear Algebra Appl., 32, (1980), 33-48. E203 
[15] N. J. Higham, Test matrix toolbox, MATLAB 4.2c User contributed M-Files,

http://www . mathworks. com/support/ftp/linalgv4. shtml. E190

[16] W. D. Hoskins and P. J. Ponzo, Some properties of a class of band matrices, Maths. Comp., 26, (1972), 393-400. E206

[17] G. Y. Hu and R. F. O'Connell, Analytical inversion of symmetric tridiagonal matrices, J. Phys. A:Math. Gen., 29, (1996), 1511-1513. E190

[18] M. Kac, W. L. Murdock and G. Szegö, On the eigenvalues of certain Hermitian forms, J. Rat. Mech. Anal., 2, (1953), 767-800. E186, E189

[19] L. Losonczi, Eigenvalues and eigenvectors of some tridiagonal matrices, Acta Mathematica Hungarica, 60, (1992), 309-322. E188, E201

[20] G. Meurant, A review on the inverse of symmetric tridiagonal and block tridiagonal matrices, SIAM J. Matrix Anal. Appl., 13, (1992), 707-728. E187, E202

[21] L. Rehnqvist, Inversion of certain symmetric band matrices, BIT, 12, (1972), 90-98. E189

[22] P. A. Roebuck and S. Barnett, A survey of Toeplitz and related matrices, Int. J. Systems Sci., 9, (1978) 921-934. E186

[23] F. Romani, On the additive structure of the inverses of banded matrices, Linear Algebra Appl. 80, (1986), 131-140. E190 
[24] P. Rozsa, On the inverse of band matrices, Integ. Eqns Oper. Theory, 10, (1987), 82-95. E187, E190, E199

[25] P. Rozsa, F. Romani, On periodic block-tridiagonal matrices, Linear Algebra Appl., 167, (1992), 35-52. E201

[26] D. E. Rutherford, Some continuant determinants arising in physics and chemistry, Proc. Royal Soc. Edinburgh, Sect A, 62, (1947), 229-236. E188

[27] G. Szegö, Solutions to problem 3706 (Proposed by Raphael Robinson) American Mathematical Monthly, 43, (1936), 246-259. E188

[28] J. Todd, The problem of error in digital computation, in Error in Digital Computation, Vol 1 ed L. B. Rall (Wiley 1965), 31 E187, E188, E196

[29] O. Toeplitz, Zur transformation der Scharen bilinearer Formen von unendlichvielen Veranderlichen, Nachrichten der Kgl. Gesselschaft der Wissenschaften zu Göttingen, mathematisch-physikalische Klasse, (1907), 110-115. E186

[30] W. F. Trench, Properties of some generalizations of Kac-Murdock-Szegö matrices, Structured Matrices in Mathematics, Computer Science and Engineering II (Boulder, CO, 1999) 233-245, Contemp. Math. 281, Amer. Math. Soc., Providence, RI, 2001 E193

[31] W. F. Trench, An algorithm for the inversion of finite Toeplitz matrices, SIAM J., 12, (1964), 515-522. E207

[32] W. F. Trench, On the eigenvalue problem for Toeplitz band matrices, Linear Algebra Appl., 64, (1985), 199-214. E207 
[33] W. F. Trench, Numerical solution of the eigenvalue problem for Hermitian Toeplitz matrices, SIAM J. Matrix Anal. Appl., 10, (1989), 135-146. E189

[34] F. Valvi, Explicit presentation of the inverses of some types of matrices, J. Inst. Maths Applics, (1977), 19, 107-117. E190

[35] H. A. Yamani and M. S. Abdelmonem, The analytic inversion of any finite symmetric tridiagonal matrix, J. Phys. A:Math. Gen., 30, (1997), 2889-2893. E190 